\title{
Observations of asteroids.
}

\begin{tabular}{|c|c|c|c|c|c|c|c|c|}
\hline Object & Date & Gr. M. T. & & $\alpha$ & $\delta$ & \multicolumn{2}{|c|}{ approx. daily motion } & Magn. \\
\hline I910 KG (new) & I 9 IO May 6 & $15^{\mathrm{h}} 24^{\mathrm{m}} 3 \mathrm{O}^{\mathrm{s}}$ & I 4 & $48^{m} 4^{6} \cdot 4$ & $-0^{\circ} 28.2$ & $-50^{\circ}$ & $-2^{\prime}$ & $\mathrm{I} 3^{\mathrm{m}}$ \\
\hline I9 10 KH (new) & 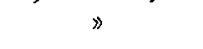 & 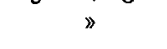 & 14 & $5^{\circ} \quad 3^{6.4}$ & +34.7 & -50 & -3 & I 3 \\
\hline$(600)[1906 \mathrm{UM}]$ & $»$ & $»$ & I 4 & $5^{8} \quad 9.4$ & to 32.6 & & & $13 \cdot 5$ \\
\hline rg Io KJ (new) & 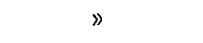 & $»$ & I 4 & 59 I $7 . \mathrm{I}$ & -o 5.4 & -50 & +5 & $13 \cdot 5$ \\
\hline (28) Bellona & $»$ & $»$ & I 5 & $03^{\mathrm{T}} . \mathrm{I}$ & -2 & & & \\
\hline I9 I0 KK (new) & $》$ & $\nu$ & I 5 & I 58.2 & $+\mathrm{I} 3 \mathrm{I} . \mathrm{I}$ & -50 & +3 & I 4 \\
\hline I9 ro KL (new) & $》$ & $»$ & I 5 & 538.3 & to 32.1 & -50 & +2 & I 3 \\
\hline
\end{tabular}

The planets were photographed on one plate with a double exposure, but the weather has been so cloudy here, that they could not be taken again. The daily motions are eye estimates.

Taunton, Mass., r 9 ro June 23 .

Foel H. Metcalf.

\section{Une variable nouvelle 42.1910 Draconis.}

Ce 9 juin Mme. L. Ceraski a trouvé une variable nouvelle dont les coordonnées approchées sont:

$$
\begin{aligned}
& \alpha={ }_{1} 8^{\mathrm{h}} 47^{\mathrm{m}} 42^{\mathrm{s}} \quad \delta=+48^{\circ} 44^{\prime} \quad(\mathrm{I} 855.0) \\
& =\begin{array}{lll}
184853 & =+4847 \quad \text { (1900.0) }
\end{array}
\end{aligned}
$$

La discussion de 26 clichés obtenus entre 1900 et 1909 fait voir que l'éclat de cette étoile varie de $10^{\mathrm{m}}$ à $\left\langle\mathrm{r}^{\mathrm{m}}\right.$. Il n'y a que quatre clichés sur lesquelles la variable est visible. M. Blažko ne peut pas dire avec certitude quel en est le type.

I 9 ro iuin $16 / 29$.

Prof. W. Ceraski.

\section{Todesanzeige.}

Am I 2. Mai d. J. verstarb nach nur eintägiger Krankheit im hohen Alter von 86 Jahren der Mitbegründer der Astrophysik, Sir William Huggins. Sein Tod hat eine schwer auszufüllende Lücke gerissen, denn bis in seine letzten Jahre war bei völliger geistiger Frische Huggins eine Autorität, deren Rat stets ein sehr beachtenswerter und maßgebender war.

William Huggins wurde in London am 7. Februar 1824 geboren. Nachdem er die City of London School absolviert hatte, schlug er nicht ein geregeltes Universitätsstudium ein, sondern ließ sich von Privatlehrern in denjenigen Fächern unterrichten, die seinen wissenschaftlichen Veranlagungen und Neigungen entsprachen. Diese aber waren sehr vielseitig. Er studierte die klassischen und modernen Sprachen, Mathematik und Naturwissenschaften, speziell Chemie, Elektrizität und Astronomie. Endlich widmete er sich auch mit großem Eifer der Physiologie der Tiere und Pflanzen, so daß er eine Zeitlang schwankte, ob er sich bei Stellung seiner Lebensaufgabe dem Mikroskop oder dem Fernrohr zuwenden solle. Zum Glück für die Astronomie hat er schließlich letzteres definitiv erwählt. Er baute sich 1856 in der Londoner Villenvorstadt Tulse Hill ein Observatorium, rüstete dasselbe instrumentell gut aus und beschäftigte sich mit astronomischen Beobachtungen.

I 859 wurden $\mathrm{ihm}$ die fundamentalen Untersuchungen Kirchhoffs bekannt, und sofort entschloß er sich, dieselben weiter auszubauen. Seine eigenen Worte über diese wichtige Epoche seines Lebens sind: 》The news was to me like the coming upon a spring of water in a dry and thursty land. Here at last presented itself the very order of work for which in an indefinite way I was looking. A feeling as of inspiration seized me. I fell, as if I had it now in my power to lift a veil, that never before had been lifted; as if a key had been put into my hands which would enlock a door which had been regarded as for ever closed to man. " Und nun folgen seine, zuerst mit Professor Miller erhaltenen Resultate auf dem Gebiete der Sternspektroskopie, bis 1864 die wichtigste seiner Entdeckungen, die Auffindung von wirklichen Gasnebeln am Himmel, den schon bekannten Astrophysiker zum berühmten Mann machte, der bis zu seinem Lebensende die sämtlichen Zweige der Astrophysik mit großem Erfolge bearbeitet hat.

Es ist hier nicht der Raum, auch nur annähernd seine verschiedenen Untersuchungen anzufïhren oder gar zu beleuchten; sie sind allgemein bekannt und jetzt leicht zugänglich, nachdein seine wissenschaftlichen Werke in zwei Bänden 1899 und 1909 erschienen sind; es mögen daher nur einige ganz allgemein gehaltene Bemerkungen darüber gegeben werden.

Vielfach ist die Ansicht verbreitet, daß es diejenigen, die zuerst in ein neues Gebiet der Wissenschaft eindringen, im Verhältnis zu ihren Nachfolgern sehr leicht haben, daß die wichtigsten Entdeckungen sich gleichsam. von selbst darbieten, daß z. B. ein Blick in das auf einen Nebelfleck gerichtete Spektroskop die 